\title{
Germanica
}

GERMANICA $\quad 10 \mid 1992$

Mosaïques littéraires

\section{Zeitroman et reportage : le cas de Ernst Ottwalt}

Zeitroman und Reportage bei Ernst Ottwalt

\section{Anne Lagny}

\section{OpenEdition}

Journals

Édition électronique

URL : http://journals.openedition.org/germanica/2093

DOI : 10.4000/germanica.2093

ISSN : 2107-0784

\section{Éditeur}

Université de Lille

\section{Édition imprimée}

Date de publication : 1 janvier 1992

Pagination : 97-110

ISSN : 0984-2632

\section{Référence électronique}

Anne Lagny, «Zeitroman et reportage : le cas de Ernst Ottwalt », Germanica [En ligne], 10 | 1992, mis en ligne le 06 février 2014, consulté le 06 octobre 2020. URL : http://journals.openedition.org/germanica/ 2093 ; DOI : https://doi.org/10.4000/germanica.2093

Ce document a été généré automatiquement le 6 octobre 2020.

(C) Tous droits réservés 


\title{
Zeitroman et reportage : le cas de Ernst Ottwalt
}

\author{
Zeitroman und Reportage bei Ernst Ottwalt
}

Anne Lagny

1 Le Zeitroman, le roman d'époque, est un genre où la question de l'achèvement de la forme touche essentiellement à celle du temps. De ce point de vue, il se situe entre deux autres genres qui représentent deux pôles contraires: le reportage et le roman historique. Défini comme roman, composition, le Zeitroman présente des affinités avec le roman historique, mais il s'en distingue néanmoins par le caractère inachevé de l'époque qu'il veut figurer. Roman de l'époque ouverte, il s'oppose au roman historique dont l'action se situe dans une époque révolue, c'est-à-dire close, et dont le narrateur est encore souverain, ordonnateur de la réalité. L'auteur de Zeitroman, lui, encore immergé dans la confusion de l'époque contemporaine, paraît plus proche à cet égard du journalisme qui fait œuvre d'information. Il utilise en principe les mêmes matériaux, qui lui sont fournis par l'actualité. Nous négligerons ici les rapports avec le roman historique pour étudier la parenté avec le reportage.

2 Proche du reportage, voire à l'occasion contaminé par ses méthodes, le Zeitroman de la République de Weimar se distingue des grands romans consacrés à l'évocation de l'avant-guerre comme une époque révolue, ceux de Thomas Mann et de Musil, par exemple. En prise directe sur l'actualité, il en garde un caractère limité, fragmentaire.

3 L'évidente proximité du journalisme donne au Zeitroman de la République de Weimar sa physionomie caractéristique par rapport au Zeitroman tel qu'il se réalise à d'autres époques. L'essor du journalisme est un fait culturel marquant de cette époque, ne serait-ce que par la réflexion qu'il suscite sur le renouvellement des techniques du récit à l'ère de la communication de masse ${ }^{1}$. On voit apparaître dans les romans la thématisation de l'information, sa transparence, son opacité dans des affaires, des procès ${ }^{2}$.

4 Est-ce à dire pour autant que le journalisme fournisse à un romancier une forme porteuse? Il semble qu'en bien des endroits, le Zeitroman réfléchisse la conscience 
d'une limite, au-delà de laquelle il serait purement et simplement réductible au reportage. Georg Lukacs, marqué dans sa réflexion par les normes d'une esthétique classique, a été immédiatement sensible au bouleversement introduit dans le genre romanesque traditionnel par l'irruption, avec le reportage, d'un nouveau mode de récit, selon lui étranger à la figuration, essentielle au roman. Dans sa célèbre controverse avec Ottwalt, à propos du roman Denn sie wissen, was sie tun, il radicalise l'opposition entre la technique du reportage et celle du roman ${ }^{3}$. Dans le reportage, le trait le plus remarquable est la place qu'y tient l'établissement des faits, la reconstitution de l'affaire, la découverte des matériaux, d'autant plus que la réalité à découvrir est dissimulée par ceux qui ont intérêt à la travestir ou à ne pas la laisser filtrer ${ }^{4}$. Le reportage, soucieux de véracité et d'authenticité, se construit par le montage de documents. Dans la conclusion, le reporter intervient pour dégager la signification d'un cas reconnu comme typique, au terme d'une analyse méthodique. En soulignant l'exemplarité du cas, le reporter donne à ses considérations la portée d'un jugement sur un phénomène d'époque. A ce point, l'engagement du reporter est déterminant ; dans sa prise de position sans équivoque, culmine la logique démonstrative caractéristique du reportage.

5 Le plan-type du reportage est tout entier ordonné en fonction de l'intelligibilité de l'affaire. Il illustre la définition du reportage comme "méthode expérimentale» donnée par Lukacs ${ }^{5}$. La structure et récriture du reportage sont subordonnées à la volonté de démonstration de l'auteur. Néanmoins, celui-ci ne saurait se borner à la rigueur scientifique, s'il veut atteindre son objectif, qui est de sensibiliser le lecteur à la gravité des faits. L'abstraction du dossier doit être contrebalancée par la mise en œuvre d'autres techniques, le recours à certains procédés de figuration, destinés à toucher le lecteur. Sur ce point, le récit du reporter se rapproche de l'écriture romanesque, sans cependant se confondre avec elle. La différence essentielle réside dans le statut du personnage. Le type d'intervention du reporter dans des scènes dramatiques n'est pas de nature à créer l'illusion d'un personnage. Le genre du reportage, s'il peut donc intégrer certains effets dramatiques destinés à figurer le caractère spectaculaire d'une affaire, ne recherche en l'occurrence que l'impact immédiat. Il est en effet essentiel de frapper le lecteur, et le reporter ne peut y parvenir par l'exposé sec de sa documentation. Il lui faut associer le public à sa démarche pour le sensibiliser aux conclusions logiques qu'il tire de l'affaire, faute de quoi celui-ci ne pourra intégrer ces informations aux cadres de son expérience. Il lui faut montrer la recherche de la vérité en action pour le tirer de son indifférence, et par là de son ignorance, et lui faire percevoir par des moyens dramatiques un enjeu en dernière instance politique ${ }^{6}$.

6 Ernst Ottwalt ${ }^{7}$ est le romancier dont les œuvres se rapprochent le plus de l'esprit du reportage, et en ce sens, le novateur le plus radical de la technique romanesque. C'est chez lui que la part faite à la documentation, aux faits véridiques est la plus importante.

7 Dans sa préface à son œuvre la plus achevée, Denn sie wissen, was sie tun ${ }^{8}$ Ottwalt écrit : «Ce livre n'est pas un roman à clés. Pourtant, le personnage (Figur) du juge Friedrich Wilhelm Dickmann n'est une création de l'imagination (Phantasieprodukt) que dans la mesure où je ne lui ai pas prêté les traits d'un juge allemand précis. En revanche, toutes les affaires judiciaires, les procès, les jugements, les événements mis ici en lumière sont des faits qui se sont produits dans les années 1920-1931, et en tant que tels, vérifiables. L'évocation $\mathrm{du}$ fonctionnement interne de la justice allemande repose elle aussi entièrement sur des faits $»^{9}$. Dans la suite de sa préface, Ottwalt invite le lecteur 
sceptique à consulter l'ample documentation qu'il a constituée sur le sujet ${ }^{10}$. La véracité des faits est la condition et la garantie de la portée du roman. Le recours partiel - nous reviendrons à la part de fiction que réserve l'auteur dans son roman - à des techniques journalistiques, est à mettre en relation avec le but que se propose Ottwalt : l'impact immédiat sur le lecteur.

8 La carrière du juge Dickmann, le juriste moyen, un homme médiocre, mais non foncièrement mauvais au départ, apparaît comme « le produit de son éducation, de sa caste, du système $»^{11}$. L'œuvre de Ottwalt, l'un des plus vigoureux réquisitoires contre la Klassenjustiz écrits sous la République de Weimar, reproduit les conclusions essentielles de l'analyse du social démocrate Ernst Fraenkel ${ }^{12}$. La justice apparaît ici comme un instrument du pouvoir, au service de la classe dominante dans son combat contre le prolétariat. La démonstration qui en est faite met en évidence, à chaque étape, la connexion entre l'idéologie et l'appartenance de classe telle qu'elle se définit économiquement et sociologiquement. Les juges, qui se recrutent presque exclusivement dans les couches de la haute bourgeoisie, formaient à la fin de l'Empire allemand un corps de fonctionnaires jouissant d'un certain prestige social ${ }^{13}$, dévoués à l'État et attachés à la forme monarchique. Le père de Dickmann est le représentant typique de ces juges dont la situation est ébranlée après la guerre, du fait de l'établissement de la République et de la grande dépression économique qui sape leur aisance matérielle, ils sont hantés par le spectre du déclassement. La caste des juges est donc acculée à prendre une attitude défensive, contre la République et contre le prolétariat, accusé d'être le principal responsable de la destruction de l'ordre social et de la corruption des valeurs morales. L'attitude des juges, leur partialité évidente dans les procès, sont conditionnées par leur mentalité réactionnaire et leur " ressentiment ", dont les ouvriers, tout particulièrement, sont les victimes. Le réflexe de classe joue en faveur du jeune Dickmann et lui permet de gravir sans encombre les échelons successifs de la carrière de juge, en écartant les tendances centrifuges, en banalisant ses doutes personnels et ses crises. C'est ce mécanisme qui est dénoncé par Ottwalt. Car si la mentalité réactionnaire de Dickmann n'est, en définitive, que le produit d'un système, ce conditionnement fait de lui, à la fin, l'instrument d'un pouvoir répressif qui juge et qui tue. Dickmann, le représentant de sa classe, doit être condamné pour les actes qu'il a été amené à commettre.

9 C'est selon le principe de gravité croissante de la faute que se construit la progression du roman. Dans la première partie, qui coïncide avec les années de formation et de stage du jeune juge, Ottwalt met en place les rouages du mécanisme dans lequel Diekmann se trouve pris. A la fin de la Première Guerre mondiale, Dickmann se retrouve désorienté par le cours des événements, comme tous les vétérans ${ }^{14}$, mais sa mentalité, héritée de son milieu, lui fait voir dans la révolution le crime impuni, et dans l'ordre ancien l'incarnation de la vraie «justice». Ses années d'études reproduisent le parcours obligé du jeune bourgeois: entrée dans le "Corps Markomannia Jena", première étape de l'intégration sociale et garantie pour l'avenir ; liaison éphémère avec une jeune fille de condition modeste, qu'il abandonne dès qu'il apprend qu'elle est enceinte de lui - on pense ici à Heinrich Mann - ; études médiocres. Lors du putsch de Kapp, Dickmann est impliqué dans l'exécution sommaire d'ouvriers communistes, mais acquitté avec ses camarades au procès de Marbourg. Durant son stage, Dickmann voit se consommer le divorce entre la justice et le droit manipulé par des juristes peu scrupuleux. C'est sa première expérience de la justice de classe, qui le laisse démoralisé. Lors du fameux procès de la Tchéka ${ }^{15}$, Dickmann est heurté par la révélation du 
fonctionnement de la machine judiciaire. Sa révolte contre son père est étouffée. Il est envoyé comme juge au tribunal de première instance de Pörgelau. Un épisode crucial s'insère dans les deux chapitres qui précèdent l'entrée de Dickmann dans la vie active, et coïncident avec une période de total désarroi : la rencontre et la liaison éphémère avec une jeune stagiaire, communiste, Genia Lazar. Dickmann dispose à ce moment d'une certaine marge de manœuvre, plus théorique que réelle, il est vrai, dans la mesure où ses opinions, ses principes, sa personnalité sont déjà façonnés par son milieu et son éducation. Pour l'essentiel, il est déjà passé insensiblement de la justice (Gerechtigkeit) à la loi (Gesetz) de l'idéal abstrait, reflet de sa mentalité réactionnaire, mais aussi d'un sens confus de la légalité, à la brutalité de la pratique quotidienne. A ce stade, la défense de principes n'est qu'un substrat idéologique destiné à masquer les intérêts matériels d'une classe. Genia le place devant un défi humain et intellectuel. Elle démasque en lui la "personnalité autoritaire ", en même temps qu'elle lui donne une clé de compréhension de sa situation. Pour un bref moment, Dickmann n'est plus totalement protégé par le code de conduite et le système idéologique de son milieu, il dévoile les contradictions et les failles de sa position. Les rencontres avec Genia coïncident avec les crises de Dickmann, la dernière à l'occasion du procès de la Tchéka. La confrontation unique entre le représentant de la mentalité réactionnaire et la jeune communiste est l'épreuve de vérité. A l'instant où Dickmann va basculer définitivement du côté de la réaction, les révélations de Genia, si elles n'ont pas pour fonction d'éclairer Dickmann, si elles sont inassimilables pour la mentalité réactionnaire, instruisent le lecteur en le plaçant, déjà, devant les conséquences futures que la deuxième partie du roman viendra confirmer. Les actes qu'il va poser sont prévisibles dans la logique du système et condamnables. Le personnage de Genia lève les doutes, formule avec une clarté définitive l'alternative : rester dans le système et se faire le complice de la répression; accepter les périls du combat pour la vérité et la justice. La fonction essentielle du roman, l'éducation politique et l'appel à l'engagement, est confiée à un personnage de femme, juive et communiste.

La suite du roman montre Dickmann dans l'exercice de ses fonctions, investi de responsabilités de plus en plus lourdes, jusqu'au moment où le dernier pas est franchi : la signature d'un arrêt de mort. Envoyé par les soins de son père dans un milieu sûr, fief de la réaction, tenu par les Junkers, Dickmann, comme Diederich Heßling, une génération auparavant, fréquente les milieux de l'aristocratie provinciale. Par là-même, il consacre son alliance avec une classe qui affiche de manière illégale ses sympathies monarchistes, cautionne la politique patronale de répression à rencontre des ouvriers, et utilise la machine judiciaire pour la défense de ses intérêts particuliers. De plus en plus, Dickmann est impliqué comme juge dans des affaires où sont manifestement favorisés les représentants de l'ordre établi au détriment des pauvres. La même société qui tolère l'adultère et la dépravation des mœurs condamne à mort un vagabond jugé pour crimes passionnels et la sentence est exécutée au moment même où l'on débat en Prusse de l'abolition de la peine capitale. Momentanément ébranlé par l'exécution de la sentence, Dickmann retourne à Berlin pour y poursuivre sa carrière de juge d'instruction. Dépassé par les problèmes de l'époque (campagne contre le \$218, scandale des conditions de la détention préventive, misère de l'institution carcérale, chômage, délinquance), il se résigne à ne plus penser au-delà de l'horizon borné de sa routine quotidienne. «Icare atterrit sur un tas de fumier ».

11 Lukacs, représentant d'une conception classique du roman, reconnaît au romanreportage le mérite de se détourner du psychologisme de l'époque précédente et de se 
concentrer sur un « contenu purement social $»^{16}$. Mais fondamentalement, il reproche à Ottwalt d'insister exclusivement sur le contenu, de manière " mécanique, unilatérale ${ }^{17}$. Selon lui, le romancier se borne ici à démasquer des mécanismes, en énonçant des jugements de valeur de nature morale. Il échoue à «figurer » (gestalten) véritablement les conflits sous la forme d'un réseau de relations entre des personnages qui seraient fortement individualisés tout en restant typiques, c'est-à-dire représentants exemplaires d'une classe sociale. Pour Luckas, le roman de Ottwalt n'est rien d'autre qu'une "pure expérience formelle: la tentative de renouveler le roman par les méthodes du journalisme ${ }^{18}$. En réponse à ces critiques, Ottwalt dénonce les limites de la position de Lukacs, prisonnière d'un esthétisme dépassé. Pour Ottwalt, il s'agit de changer la conscience du lecteur, et non de le bercer dans la jouissance esthétique d'une belle totalité. Son roman se proclame fonctionnel, immédiatement convertible en termes d'action et non de contemplation ${ }^{19}$. Le lecteur est pris à témoin dans le procès de la justice de son époque.

Ce que le reportage, dans sa rapidité, ne permet pas, le roman de Ottwalt se donne les moyens de le faire. Dans sa préface, l'auteur reconnaît implicitement que son roman de la justice sous la République de Weimar ne peut se passer d'une part de fiction, qui se trouve justement coïncider avec la création d'un personnage ${ }^{20}$. Le travail de l'auteur a consisté à dépouiller le juge-type Dickmann de tous les traits individuels susceptibles de favoriser une identification avec tel ou tel juge particulier, qui priverait le personnage de sa dimension absolument typique, exemplaire.

Le juge au nom pompeux, autant qu'évocateur (la tradition conservatrice est ici alliée à la suggestion de la médiocrité du personnage) figure comme accusé. L'auteur le convoque devant un tribunal fictif au moment même où il est décrété coupable: l'instant - c'est le premier chapitre du roman - où l'injustice à laquelle il participe ne l'empêche plus de dormir, où il a fini par s'installer dans une bonne conscience factice, durement gagnée au terme d'une «formation» conditionnée dès le départ par son milieu et son appartenance de classe ${ }^{21}$.

Le but du réquisitoire prononcé par l'auteur - il y a bien, dans ce roman, quelque chose qui ressortit à la communication orale, à la rhétorique du plaidoyer - est de montrer comment, à certains moments significatifs, aux carrefours qui jalonnent sa carrière, le personnage s'engage dans une voie tracée d'avance. Comment les virtualités, les aspirations individuelles sont étouffées par la logique des rapports de force. L'homme sans envergure personnelle, le médiocre tel qu'il s'incarne dans le personnage de Dickmann, est "destiné », comme produit de son milieu, à devenir l'un des rouages de la machine judiciaire qui écrase et tue, derrière le masque d'une légalité spécieuse, les adversaires politiques, les petites gens, les ouvriers. Toute tentative de sa part d'échapper à cette détermination n'a aucune chance d'aboutir à un renversement de situation, parce que Dickmann est impuissant à réfléchir ce qui le conditionne, et que ses velléités même de réflexion le mèneraient à une impasse idéologique et pratique à l'intérieur du système. En effet, celui-ci le protège, lui épargne tout retour sur luimême, gomme les conséquences de ses actes répréhensibles. Prisonnier, otage du système, Dickmann ne s'y maintient qu'au prix d'une adaptation forcée qui se traduit par une insensibilisation progressive à toute conscience, tant morale que politique, et lui coûte quelques dysfonctionnements, banalisés en troubles psychologiques.

Le roman de Ottwalt apparaît ici comme la caricature du Bildungsroman, centré sur l'intériorité du personnage, et qui intègre les données du monde extérieur, les 
rencontres, les voyages... en les convertissant en expérience intérieure, en les unifiant dans la perspective de la croissance organique d'une personnalité singulière. L'auteur épouse le plus étroitement possible la dynamique de l'épanouissement individuel. La carrière du juge Diekmann, type social, doit être comprise comme antiformation ${ }^{22}$. Loin de se développer, l'individu s'atrophie. Ce sont les puissances extérieures, la société qui prennent le dessus. Nous sommes ici au stade extrême d'un processus de destruction du Bildungsroman - c'est-à-dire de l'individu - dont une phase intermédiaire serait marquée par le roman de Heinrich Mann, Der Untertan : dans ce dernier cas, le concept de l'individualité est vidé de sa substance, dans la mesure où le personnage de Diederich Heßling - qui annonce par bien des traits celui du juge Diekmann - est tout entier réductible à la mimicry. Le lecteur assiste encore néanmoins à la formation d'une personnalité, aliénée, inauthentique: le héros de Heinrich Mann s'affirme comme histrion. Le centre de gravité du roman se déplace ici de la figuration de l'intériorité de l'individu vers le contenu social, néanmoins l'œuvre de Heinrich Mann repose encore sur la possibilité d'identification au personnage - fût-ce dès lors de manière ironique et distanciée. Les liens étroits qui se tissent, au cours du roman, entre Diederich, qui s'affirme comme représentant du pôle de la puissance (Macht), et Buck, acteur de la révolution de 1848, ainsi que son fils, Wolgang Buck, tous deux incarnant le pôle de l'esprit (Geist), interdisent, par leur complexité, leur indication, de réduire le personnage de Diederich à une silhouette sans épaisseur, et donnent à l'affrontement brutal entre les deux principes une véritable ampleur dramatique.

Dans le roman de Ottwalt, en revanche, l'intériorité du personnage subsiste à l'état de simple trace, notamment dans le débat entre les aspirations personnelles et les impératifs de classe. Le projet du roman ne consiste pas à suivre l'évolution intérieure du personnage (comme chez Heinrich Mann encore, dont le roman s'ouvre sur l'enfance de Diederich ${ }^{23}$ ), mais à montrer par quel enchaînement le juge Dickmann est arrivé au moment où il est décrété coupable. La normalisation de Dickmann, qui se transforme en fonctionnaire totalement adapté aux exigences de son rôle, consacre l'écroulement du personnage, et cette abdication définit son crime. Aux différentes stations de son itinéraire, il a refusé de faire l'effort d'une prise de conscience. Le titre du roman est sans équivoque « ils savent ce qu'ils font».

Dans la logique propre au réquisitoire, le procureur général n'est pas tenté d'encourager dans l'auditoire une quelconque tentation d'identification avec l'accusé. Bien au contraire, l'ensemble des moyens rhétoriques mis en œuvre doivent concourir à exclure d'emblée cette possibilité. En revanche, il doit représenter avec force la gravité du crime pour gagner l'auditoire à l'idée d'un nécessaire châtiment. S'il peut évoquer le conflit intérieur chez l'accusé, ce n'est pas dans le but d'éveiller une sympathie qui suspendrait le jugement. Il doit présupposer que l'accusé avait la faculté et la possibilité de choisir de ne pas être coupable, et donc écarter les éléments qui fonctionneraient comme circonstances atténuantes ${ }^{24}$.

L'engagement de Ottwalt, qui dénonce en la personne du juge Dickmann le scandale de la justice de classe sous la République de Weimar, contraste fortement avec le point de vue éclairé, souverain d'un Heinrich Mann, qui marque par l'ironie, la caricature, ses distances vis-à-vis du personnage de Diederich, sans le désigner comme l'ennemi de classe à abattre. On voit ici ce qu'apporte au roman de Ottwalt la méthode journalistique : la logique percutante de la démonstration (qui représente un raccourci, une réduction de ce qui serait déployé et nuancé dans un roman traditionnel), l'impact 
d'une actualité brûlante. Le recours à la forme romanesque représente une amplification et une intensification de l'effet produit par le reportage, il mobilise et oriente l'indignation du lecteur non pas contre une institution, mais contre ses représentants. Le personnage de Diederich Heßling n'est pas condamnable au sens propre du terme dans la perspective de Heinrich Mann, c'est le type humain et social dans lequel une époque peut reconnaître son visage. Le juge Dickmann, lui, est reconnu coupable avec préméditation et condamné sans appel.

19 Pour Ottwalt, que son engagement communiste rapproche des auteurs de romans ouvriers comme Willy Bredel, l'œuvre écrite est un relais de l'action politique, dans un contexte général marque par l'urgence. La part de figuration romanesque est réduite au minimum (de trop larges concessions à la fiction ne pourraient qu'affaiblir la force d'impact directe du livre) et subordonnée à une logique démonstrative qui impose une très forte schématisation pour rendre typiques les personnages et les processus décrits. Les faits retenus pour leur exactitude documentaire prennent leur pleine signification en tant que séquences d'un processus dénoncé comme pervers. A la lumière de l'analyse politique, le roman construit un modèle d'explication. La lisibilité de l'époque est un enjeu majeur pour l'auteur dans la perspective de l'engagement militant. «L'ensemble des rapports sociaux s'est compliqué à tel point dans la conscience des hommes qu'il faut résoudre cette complexité $»^{25}$. S'il est impossible de prendre une vue générale de l'état de la société, il importe néanmoins d'éclairer le lecteur en faisant apparaître, à travers une réalité opaque, la logique du monde à l'envers. Le souci d'efficacité pragmatique conditionne le découpage et l'isolement de «complexes de faits ${ }^{26}$ choisis pour leur lisibilité. Ces Tatsachenkomplexe - la carrière d'un juge, comme la lutte entre les ouvriers et le patronat dans le roman de Bredel Maschinenfabrik N\&K sont le matériau romanesque par excellence. Le rétrécissement du champ de vision est compensé par l'évidence exemplaire de l'analyse.

20 Le cas limite de Ernst Ottwalt illustre une conception particulière de la littérature affranchie de sa finalité purement esthétique pour les besoins de la lutte des classes. D'une réalité politique, il s'agit de tirer des conséquences non seulement esthétiques, mais pratiques.

On reconnaît sans peine la similitude entre les conceptions esthétiques du romancier communiste Ottwalt et celles de John Heartfield dans le domaine de la photographie. Le photomontage, au principe duquel on trouve l'analyse politique, procède à la manière de l'écrivain isolant d'une réalité opaque des Tatsachenkomplexe. Dans ce type de photographie, des éléments sont d'abord sectionnés, découpés, puis assemblés de manière à faire jaillir une signification occultée par le discours courant (celui de la propagande national-socialiste, par exemple). Il s'impose, à une époque où règne l'illisibilité, comme véhicule de la critique.

On trouve chez Ottwalt comme chez Heartfield, la même réduction schématique, le même montage efficace, caractéristiques d'une esthétique fonctionnelle au service de l'engagement politique immédiat. Le rapprochement avec le photomontage présente l'intérêt de faire saisir encore plus nettement la nature de l'efficacité de ces formes d'œuvre d'art. En effet, le photomontage, par l'utilisation de la photographie, et non pas du dessin, ou de la peinture, supprime tout détour dans la trajectoire qui vise à atteindre sa cible. Il est beaucoup plus immédiat que la caricature. Ce n'est pas un hasard si les dénonciations les plus violentes de Hitler sont justement les œuvres de Heartfield plutôt que des caricatures. Celle-ci déréalise son objet, alors que le 
photomontage, de par son support même, le rend aussi fortement présent que possible, aussi vrai que nature, pour l'attaquer de front. Ce maintien d'un contact étroit avec une base documentaire authentique (la photographie, chez Heartfield, le dossier constitué sur les affaires judiciaires contemporaines, chez Ottwalt) exclut la possibilité de prendre ses distances vis-à-vis de ce qui nous est montré. Ottwalt et Heartfield assènent la vérité des rapports sociaux comme un coup, et la violence du choc, loin d'être amortie, est au contraire décuplée par la puissante synthèse logique du montage photographique ou romanesque, qui matérialise une thèse. La loi des dysfonctionnements sociaux, cachée dans la réalité quotidienne confuse, ou intentionnellement recouverte par une idéologie mensongère, est rendue instantanément lisible.

\section{NOTES}

1. - On consultera sur ce point, par exemple, les textes de Benjamin consacrés à l'écriture journalistique et au mode spécifique de perception dégradée de la réalité qu'il implique, cf. "Quelques thèmes baudelairiens », in Euvres choisies, tome 1, Paris, Julliard, 1959, p. 241-242 (trad. fr. Gandillac).

2. - Que l'on pense ici par exemple au procès de Neumünster, transposé dans le roman de Fallada, Bauern, Bonzen und Bomben, et dans celui de Ernst von Salomon, La Ville; à la dénonciation des manœuvres du patronat pour s'assurer la mainmise sur les organes de presse dans Union der festen Hand, de Erik Reger... De façon générale, le Zeitroman utilise les événements couverts par le journalisme, il se fait l'écho d'une actualité spectaculaire. Précisons que la génération d'écrivains dont les débuts coïncident avec la crise économique sont presque tous, à l'origine, journalistes (Reger, Fallada, Gabriele Tergit...). Cf. Michael Winkler, «Paradigmen der Epochendarstellung in Zeitromanen der jüngsten Generation Weimars», Weimars Ende, ed. Thomas Koebner, Francfort / M : Suhrkamp, 1982, p. 360-375.

3. - Cette controverse a paru dans les numéros 7, 8 («Reportage oder Gestaltung» de Lukacs), 10 (Réponse de Ottwalt : «Tatsachenroman und Formexperiment») et 11-12 (Lukacs: «Aus der Not eine Tugend») de la revue Linkskurve (1932).

4. - Un exemple remarquable de la technique du reportage nous est fourni par le livre que Egon Erwin Kisch, «der rasende Reporter», le fondateur du genre du reportage sous la République de Weimar, a consacré à l'affaire Redl. La trahison de l'officier du bureau d'espionnage vendu à la Russie a été rendue publique en mai 1913 par Kisch, qui a maquillé ses révélations en " démenti » publié dans son journal. Le récit qu'il a ensuite rédigé est paru à Berlin en 1924.

5. - Pour Lukacs, le reportage relève d'une méthode scientifique : les faits présentés servent à illustrer des idées, ils ont une valeur démonstrative, alors que par la figuration (Gestaltung), le romancier restitue la dimension d'un destin individuel en tant que tel (cf. «Reportage oder Gestaltung», Linkskurve ${ }^{\circ} 7$, p. 25, et Le Roman historique).

6. - Wallraff, héritier en ligne directe de Egon Erwin Kisch, ne procède pas autrement dans ses reportages. La minutie avec laquelle il décrit sa transformation en Turc dans Ganz unten, pour ne prendre que l'exemple le plus récent, n'a d'autre but, en définitive, que d'arracher le lecteur, étranger à cette réalité qu'il côtoie sans la voir, à son indifférence, lui faire sentir, lui proposer un substrat minimum d'identification. De ce point de vue, le reportage engagé tel que le pratique 
Wallraff représente une information qualitativement supérieure à celle, abstraite et lointaine, des journaux et des bulletins de statistique : cette information est assimilable par le lecteur parce qu'elle se réfère à ses propres catégories d'expérience.

7. - L'itinéraire d'Ottwalt (né en 1901) l'amène dans sa jeunesse à s'engager du côté de la réaction, expérience qu'il thématise dans son premier roman, Ruhe und Ordnung (1929), un témoignage important sur l'impact de la démagogie nationale et sociale auprès de la jeunesse des années 20. Passé au communisme, il devient à partir de la fin des années 20 un écrivain et un publiciste extrêmement actif. Son deuxième roman, Denn sie wissen, was sie tun, écrit en 1931 et publié chez Malik-Verlag, donne lieu à l'un des débats théoriques les plus importants au sein du BPRS (Bund proletarisch-revolutionärer Schriftsteller), dont les enjeux ressortent clairement de la controverse avec Lukacs. On doit aussi à Ottwalt l'une des premières tentatives d'interprétation marxiste du fascisme allemand (Deutschland Erwache, 1932, Vielle/Leipzig). Émigré en U.R.S.S. après l'arrivée des nazis au pouvoir, il disparaît au cours des purges staliniennes. La connaissance approfondie qu'Ottwalt avait de l'idéologie fasciste, lui fait éviter l'écueil du schématisme, et donne à ses réquisitoires une grande vigueur.

8. - Nous citons le roman d'après l'édition reprint (d'après la $1^{\text {ère }}$ édition, Malik-Verlag, Berlin 1931), Verlag europäische ideen, Berlin, 1977).

9. - Op. cit., p. 7.

10. - Avec l'aide de sa femme, Waltraut Nicolas, journaliste au Deutsche Allgemeine Zeitung à la fin des années 20, spécialisée dans les chroniques judiciaires. Cf. la documentation présentée par Andreas W. Mytze en postface du roman Denn sie wissen, was sie tun, ainsi que dans un ouvrage consacré à la vie et à l'œuvre de Ottwalt.

11. - Citation de Tucholsky, dans sa recension du roman. De façon révélatrice, Tucholsky voit dans l'œuvre de Ottwalt une «aide dans le combat contre l'injustice » plutôt qu'une « œuvre artistique », et conseille à Ottwalt de se former à l'école des romanciers français !

12. - Ernst Fraenkel, Zur Soziologie der Klassenjustiz, Berlin, 1927.

13. - Le grade d'officier de réserve est l'indice de l'assimilation aux classes dominantes, pour les bourgeois, comme pour les juifs d'ailleurs.

14. - Le thème de la désorientation se retrouve dans le roman de Remarque, Der Weg zurück, qui illustre une variété de situations individuelles et de réactions personnelles, souvent poignantes, débouchant à la fin sur un engagement pacifiste, individuel et éthique plutôt que politique.

15. - Le procès de la Tchéka (février-avril 1925) est l'exemple le plus spectaculaire de la violation du droit par le «Staatsgerichtshof zum Schutz der Republik». La haute cour de sûreté de l'État, créée à la suite de l'assassinat de Rathenau, dans le cadre de la loi pour la protection de la République (21 juillet 1922), devait assurer la conduite des procès intentés aux criminels politiques. Très vite, elle fut accusée de partialité, jugeant les crimes de l'extrême droite avec indulgence, sévissant contre les mouvements d'extrême gauche. L'assassinat d'un espion de la police par Felix Neumann (chef d'un groupe terroriste clandestin, le «Bund Roter Katzen») permet d'organiser un procès dans lequel le parti communiste, interdit depuis fin 1923, devait être discrédité, et au cours duquel trois condamnations à mort furent prononcées (cf. Klaus Petersen, Literatur und Justiz in der Weimarer Republik, Stuttgart, 1988, p. 25, 111-112 ; Traditionen deutscher Justiz, Berlin, 1978, qui consacre un chapitre au procès de la Tchéka en citant des extraits des actes du procès, ainsi que des textes de Fallada, Kisch et Münzenberg, p. 201-236. On les comparera utilement avec les passages correspondants dans le roman d'Ottwalt).

16. - Cf. Linkskurve, $\mathrm{n}^{\circ} 7$, p. 25.

17. - Cf. loc. cit.

18. - Cf. loc. cit.

19. - Cf. $n^{\circ} 10$, p. 24.

20. - Cf. supra l'extrait de la préface au roman. 
21. - Ce qui ne veut pas dire que le personnage de Dickmann soit exempt d'une certaine complexité, rendue par les contradictions au niveau de la conscience du personnage essentiellement, dans la mesure où il n'a pas de véritable dimension psychologique.

22. - S'il y a «formation» de Dickmann, c'est dans le sens de la constitution d'une conscience politique, et non d'une personnalité. Cette définition du personnage s'accorde avec le genre même du Zeitroman, roman de l'époque ouverte, dont l'enjeu est justement la lisibilité de l'époque. Toutes les autres dimensions, psychologiques par exemple, sont subordonnées à la figuration de la problématique de l'époque, du sens de l'époque encore à construire.

23. - On remarquera la différence de construction entre les deux romans : celui de Heinrich Mann épouse la genèse de la personnalité de Diederich Heßling, celui de Ottwalt s'arrête à quelques stations marquant des étapes intellectuelles, des tournants, qui supposent donc un moindre degré de figuration.

24. - Que l'on compare avec le réquisitoire de Wolfgang Buck stigmatisant pour la première fois en Diederich le type de «Untertan». Si les clivages éthiques et politiques sont perçus avec une rare clairvoyance, le brillant plaidoyer de Wolfgang Buck, qui s'apparente plutôt à la performance rhétorique, et dont les effets sont appréciés par les gens du théâtre venus l'applaudir, échoue à convaincre les acteurs politiques et n'entraîne nullement l'adhésion. Diederich Heßling, discrédité par le représentant de l'esprit, sort paradoxalement renforcé, la réalité du pouvoir qu'il incarne n'est pas affectée par cette épreuve de vérité.

25. - «Tatsachenroman und Formexperiment», Linkskurve, n¹0, p. 25.

26. - La perception du temps propre aux auteurs de romans de la modernité exclut d'emblée la saisie de grandes évolutions déployées sur une longue période; par ailleurs, le cloisonnement social étanche rend très difficile la peinture d'un véritable panorama d'époque. Le Tatsachenkomplex, le complexe de faits, est un fragment de réalité que l'on isole dans une réalité brute, chaotique, pour le construire en unité de sens. La carrière du juge Dickmann, par exemple, n'est pas le tout de la réalité de la République de Weimar, mais le roman d'Ottwalt permet de la reconstruire, dans sa logique, et de démonter ainsi le mécanisme, exemplaire, des rapports de force sous la République de Weimar. La valeur de témoignage du Zeitroman dépend donc du choix du Tatsachenkomplex, de sa capacité à réfracter la problématique d'époque.

\section{RÉSUMÉS}

Le Zeitroman utilise le même matériau que le reportage. Cet effet est particulièrement visible dans Denn sie wissen, was sie tun, de Ernst Ottwalt (surtout connu par sa controverse avec Lukacs) : véracité de la documentation, typisation des personnages. La part de la fiction consiste à construire un personnage, mais celui-ci est vu comme représentant d'une classe, et d'une idéologie, et non comme individu doté d'une intériorité. Cet anti-Bildungsroman vise à renouveler le genre romanesque par les méthodes de journalisme, et à changer la conscience du lecteur. Il est comparable, dans son efficacité, au photomontage à la manière de John Heartfield.

Der Zeitroman der Weimarer Republik greift auf denselben Stoff zurück wie die Reportage. Das läßt sich besonders gut am Beispiel von Ottwalts Justizroman Denn sie wissen, was sie tun zeigen (der direkte Anlaß zur berühten Lukacs-Ottwalt-Kontroverse): Überprüfbarkeit der Dokumentation, Typisierung der Figuren. Der Anteil der Fiktion besteht in der Gestaltung einer Figur, die aber wohl als Vertreter einer Klasse und einer Ideologie auftritt, und keineswegs als 
Individuum, das etwa durch sein Innenleben charakterisiert wäre. Insofern ist der Roman als Anti-Bildungsroman aufzufassen, der die Gattung mit den journalistischen Methoden zu erneuern versucht, und dessen Hauptanliegen die Änderung des Bewußtseins beim Leser ist. In seiner Wirksamkeit läßt er sich mit der Photomontage Heartfields vergleichen.

\section{AUTEUR}

\section{ANNE LAGNY}

Université Charles-de-Gaulle - Lille III 